\title{
Linckia multifora (Echinodermata: Asteroidea) in Rarotonga, Cook Islands: Reproductive Mechanisms and Ecophenotypes ${ }^{1}$
}

\author{
Terry 7. Crawford ${ }^{2}$ and Bruce 7. Crawford ${ }^{3}$
}

\begin{abstract}
In Rarotonga, Linckia multifora (Lamarck) exists in two forms: a blue gray type that is found on the reef intertidally and a red form that is found subtidally. Both types reproduce asexually by regeneration of autotomized arms, as well as sexually, but the relative potential for sexual reproduction varies greatly between these different sites. In the laboratory, reciprocal crosses of the blue gray intertidal form and the red subtidal form developed as successfully as the controls and were indistinguishable in morphology. In addition, both the blue gray intertidal form and the red subtidal form contain two different classes of haplotypes of the mitochondrial gene cytochrome oxidase subunit I (COI), which exhibit 12 fixed differences. These results suggest that L. multifora of Rarotonga has a dual origin and that the two different forms seen in the two environments belong to a single interbreeding population and may represent ecophenotypes.
\end{abstract}

LiNCKIA MULTIFORA (LAMARCK) is a small sea star, a member of the Ophidiasteridae, that is found on coral reefs throughout the Indo-Pacific Ocean (Clark and Rowe 1971). It generally has arms of uneven lengths because it reproduces asexually by autotomizing arms, which can then regenerate into whole starfish (Edmondson 1935). It is identified by the arrangement of spines along the ambulacral grooves, the presence of two madreporites, the arrangement of papular openings, the granularity of the epidermis, and the general shape of the arms (Clark and Rowe 1971).

On the island of Rarotonga, Cook Islands, South Pacific, there appear to be two different types of L. multifora: a red-spotted form that is found in the subtidal zone and a gray blue form that is found on the top of the

\footnotetext{
${ }^{1}$ Manuscript accepted 30 September 2006.

${ }^{2}$ Corresponding author: Departments of Zoology and Botany, University of British Columbia, 6270 University Boulevard, Vancouver, British Columbia, Canada V6T1Z4 (e-mail: tcrawfor@interchange.ubc.ca).

${ }^{3}$ Island Medical Program, University of Victoria, P.O. Box 1700, Stn CSC, Victoria, British Columbia, Canada V8V2Y2.
}

Pacific Science (2007), vol. 61, no. 3:371-381

(C) 2007 by University of Hawai'i Press

All rights reserved reef in the intertidal zone. To confirm that these are both L. multifora and to explore the relationship between them we have sequenced a mitochondria gene, cytochrome oxidase subunit I (COI).

To understand aspects of the ecology and evolutionary biology of an organism, it is important to understand its mechanism(s) of reproduction (Hart 2002). Linckia multifora is generally considered to reproduce primarily by asexual means (Rideout 1978), and it has been referred to in the literature as an asexual organism (Ebert 1996). However, it produces eggs and sperm (Mortensen 1938, Rideout 1978), and viable cultures of embryos have been produced in the laboratory (Mortensen 1938). In this study we set up breeding experiments with both types of $L$. multifora to see if viable embryos would result from both types and from both crosses. In addition, we studied the population size structure at several different sites to determine whether sexual as well as asexual reproduction occurs in nature and to determine whether the relative importance of larval recruitment varies between different sites.

\section{MATERIALS AND METHODS}

Specimens were collected from subtidal locations from depths of approximately 7 to $30 \mathrm{~m}$ 
and from intertidal locations by walking on the reef at low tide or by snorkeling. The results of these collections were recorded by photography with a size marker and color standard. Specimens to be used as a source of gametes for embryological studies were then kept in an aquarium for a few days, and specimens for DNA extraction were preserved in $70 \%$ ethanol. In some cases specimens were taken with coral rubble to Vancouver, Canada, where they were kept in an aquarium for up to $1 \mathrm{yr}$.

To set up cultures of embryos, gonads were dissected from the arms and seawater containing $0.1 \mathrm{mg} / \mathrm{ml} \mathrm{1-methyl} \mathrm{adenine} \mathrm{was}$ applied to the ovaries to cause release and maturation of the eggs as evidenced by germinal vesicle breakdown (Stevens 1970, Kanatani 1973). The mature egg suspensions of each type were washed in seawater, divided into two parts, and fertilized with diluted sperm suspension of each type. The latter was prepared by mixing one or two drops of "dry" sperm from harvested testes with $50 \mathrm{ml}$ of seawater. Each culture consisted of 100 or more fertilized eggs.

To determine whether specimens of specific sizes contained mature gonads, two approaches were used. Specimens collected in late November and early December were measured, injected with 1-methyl adenine, and if that failed to produce spawning, the gonads were isolated and treated with 1methyl adenine as already described. The presence or absence of ripe gonads was recorded for 17 red, subtidal specimens and 9 blue gray intertidal specimens. In addition seven specimens of the blue gray intertidal type that had been collected from Town Reef and preserved in $70 \%$ ethanol were measured, and about $1 \mathrm{~cm}$ of the proximal portion of the largest arm of each was sent to Wax It Histology Services, Vancouver, British Columbia, to be decalcified, embedded in paraffin, sectioned, and stained with haematoxylin and eosin. A collection of six red, subtidal L. multifora was similarly treated.

For polymerase chain reaction (PCR) studies, specimens were rehydrated overnight and chopped into small pieces. DNA was extracted according to the method of Zyskind and Bernstein (1989) with the addition of 0.5 $\mu \mathrm{g} / \mathrm{ml}$ proteinase $\mathrm{K}$ to the extraction solution. After two extractions with phenol:chloroform: isoamyl alcohol (25:24:1) and precipitation of DNA from the aqueous phase with ethanol, the DNA pellet was washed with $70 \%$ ethanol, air-dried, dissolved in distilled $\mathrm{H}_{2} \mathrm{O}$, and stored at $-80^{\circ} \mathrm{C}$.

PCR reactions were carried out with Platinum Taq DNA polymerase (Gibco) $(0.5 \mu \mathrm{l})$, $0.1 \mu \mathrm{M}$ primers, $2.5 \mathrm{mM} \mathrm{MgCl}_{2}, 0.2 \mathrm{mM}$ deoxynucleotide triphosphates, and approximately $200 \mathrm{ng}$ template DNA in a thermocycler (Applied Biosystems 2400) with the following primers for COI: LINF 5'-GCR CCR GAT ATG GCR TTY CCA-3' and LINR 5'-CCT ATW GAT ACD ATR GCR TAR ACC ATT $-3^{\prime}$.

The primers were designed from the sequence of LmWA3 (Genbank number AF 187929). The forward primer begins four bases from the start of that sequence and the reverse primer extends to four bases from the end of the LmWA3 sequence. The amplification conditions were as follows: denaturation for $3 \mathrm{~min}$ at $95^{\circ} \mathrm{C}$ followed by 30 cycles of $95^{\circ} \mathrm{C}$ for $30 \mathrm{sec}, 45^{\circ} \mathrm{C}$ for $45 \mathrm{sec}, 72^{\circ} \mathrm{C}$ for 60 sec, followed by a final extension of $10 \mathrm{~min}$.

The PCR product was electrophoresed on a $1 \%$ agarose gel in TAE. After staining with ethidium bromide the approximately 600 base pair COI fragment was recovered using an UltraClean 15 DNA Purification System kit (MOBIO) and cloned into pGEM-T Easy Vector (Promega) according to the supplied protocol. Plasmid DNA was extracted from positive colonies with a Wizard Plus Minipreps DNA Purification System kit (Promega), and the insert was sequenced using Big Dye3 Terminator Chemistry at the University of British Columbia Nucleic Acid Protein Service Unit.

Sequences were aligned with the Clustal $\mathrm{X}$ program. The aligned sequences were converted to the PAUP/NEXUS format and then analyzed with the PAUP program (Swofford 2003) using Maximum Parsimony and Neighbor Joining with the default settings, except that the maximum trees limit was set to 10,000 for Parsimony, and the Neighbor Joining distance setting was Ki- 
mura 2-parameter. The robustness of the branch points of the resulting phylograms was determined by performance of 1,000 bootstrap replicates.

\section{RESULTS}

Two morphologically different populations of L. multifora were found at various locations on and outside the fringing reef on the island of Rarotonga, Cook Islands. One type of specimen that taxonomic keys (Clark and Rowe 1971) indicated to be L. multifora was found on the top of the fringing reef at low tide. These sea stars were a dark gray blue, and some had red purple patches (Plate $\mathrm{I} A$ ). They were found in small tide pools on pebbles or partially buried among pebbles in the surf. Their sizes ranged from 1.9 to $5.3 \mathrm{~cm}$ along the longest arm with a median value of $3.5 \mathrm{~cm}$ (Figure 1A). Almost all had arms of unequal lengths, indicating that they were reproducing asexually, and comets and regenerating individuals (counter-comets, in the terminology of Rideout [1978]) of various sizes were present (Plate I $A$ ). Comets (regenerating arms) were identified as individuals having one arm at least $10 \%$ longer than the others and represented $13.2 \%$ of the specimens in Figure $1 A$.

A second type of specimen that taxonomic keys also indicated to be $L$. multifora was found during dives on subtidal reefs from depths of 3 to $30 \mathrm{~m}$. Linckia multifora was found at all locations, but their abundance varied dramatically from one subtidal reef to another. In some locations only a few individuals could be found in approximately $40 \mathrm{~min}$, but at another (known locally as Bernie's Reef) more than 50 could be found in that time. Most commonly the sea stars were found on areas of dead coral. This subtidal form of L. multifora had a cream or pale blue background with red blotches and had blue tips to the arms (Plate $\mathrm{I} B$ ). The sizes ranged from 0.9 to $5 \mathrm{~cm}$ along the longest arm with median values of 2.7 and $2.2 \mathrm{~cm}$ (Figure $1 B$ and $C$ ). Almost all had arms of unequal lengths, indicating that they were reproducing asexually. There were comets (regenerating arms) in various stages of regeneration as well as counter-comets. Some of the comets were formed from very tiny legs (less than $0.7 \mathrm{~cm}$ ) and others were regenerating from larger ones (Plate $\mathrm{I} B$ ). Of the specimens in Figure $1 B$ and $C, 13.7 \%$ and $14.9 \%$, respectively, were comets. Figure 1 also shows that the subtidal sites had the greatest frequencies of small individuals.

We considered the possibility that the blue gray form of L. multifora might be a juvenile form of L. laevigata or a hybrid of the red form of $L$. multifora with L. laevigata, a closely related species that Williams (2000) found to be indistinguishable on the basis of COI sequences. Although the blue gray $L$. multifora and the royal blue L. laevigata could sometimes be found only a few feet apart and were ripe at the same time, there were no specimens of intermediate phenotype regarding color or arm shape (pointed and irregular in length for L. multifora and even in length and rounded at the tip for L. laevigata), indicating that the blue gray form of L. multifora is not a hybrid of the red, subtidal form of $L$. multifora and L. laevigata. In addition when we examined 21 of the smallest $L$. laevigata that we could find in early December, 6 with arm lengths of 6-8 cm had immature gonads, and 15 with arms of $10-13.5 \mathrm{~cm}$ in length were all mature. In the case of the blue gray L. multifora, the largest we found had an arm $5.3 \mathrm{~cm}$ in length, and all but one of the specimens with an arm longer than $3.0 \mathrm{~cm}$ that were checked had mature gonads. Thus there is no overlap in the sizes of the mature adults of the two species, which confirms that the blue gray $L$. multifora is not an immature stage of L. laevigata. This is consistent with the observation of Marsh (1974) that on the basis of morphology L. multifora and $L$. laevigata are different species. Furthermore, Yamaguchi (1977) described the rarely seen pretransformation form of L. laevigata as characterized by distinctive brown and dark green blotches.

To confirm that both types of L. multifora were capable of sexual reproduction, in early December eggs were obtained by treating dissected gonads with 1 -methyladenine. Mature ovaries each produced several hundred eggs that were of the clear, nonyolky type. 


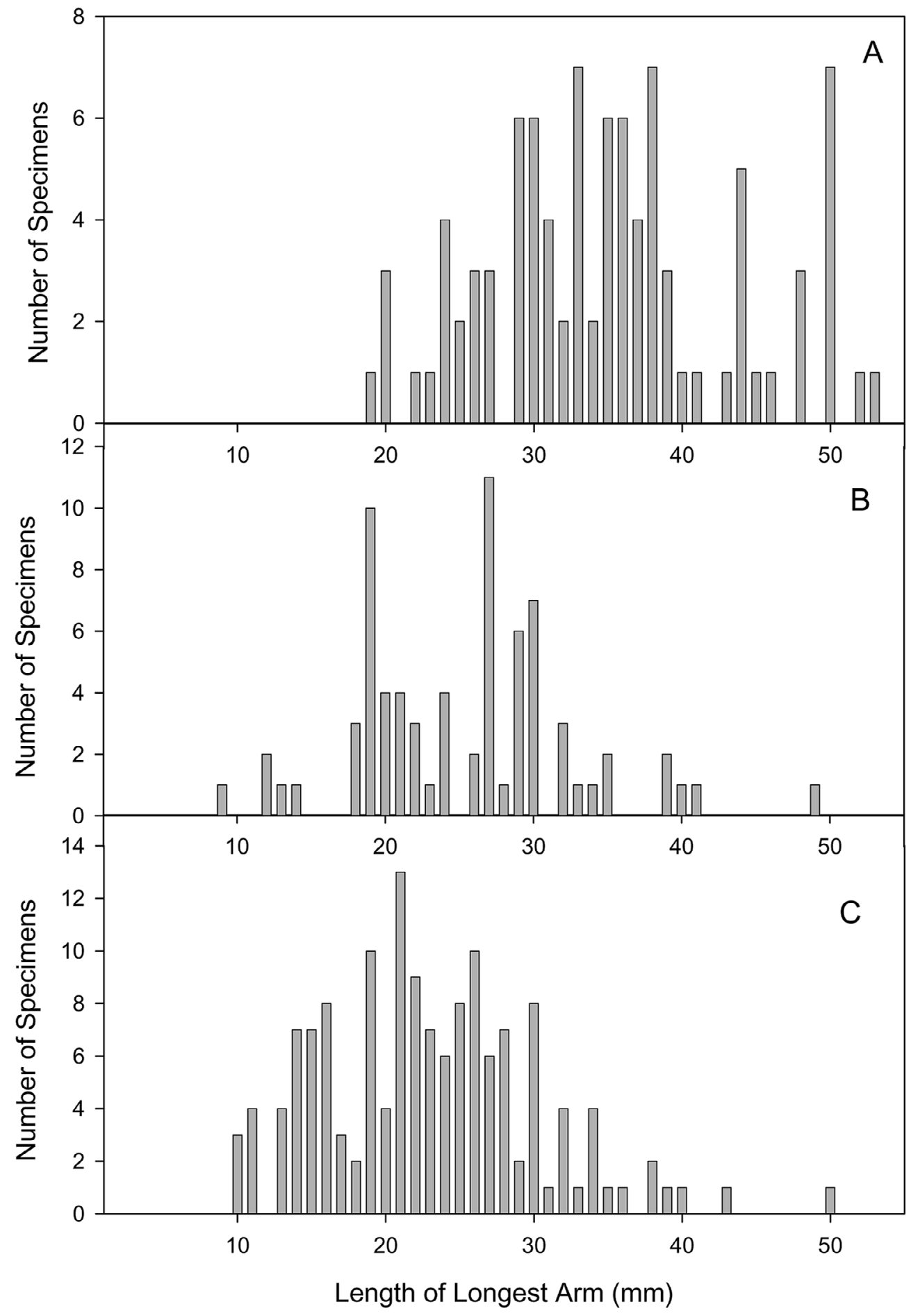

FIgURE 1. Linckia multifora. Length (mm) of longest arm of specimens from $(A)$ intertidal location (Town Reef) and subtidal locations, $(B)$ Bernie's Reef, and $(C)$ other subtidal reefs. Note the absence of very small specimens and the greater frequency of large specimens in the intertidal type $(A)$. 
After fertilization the resulting cleavage stages were typical for a planktotrophic type of echinoderm egg and resulted in a planktotrophic type of larva. Reciprocal crosses between the blue gray and red forms of L. multifora plus the controls were all set up at the same time. (This was necessary because we were unable to regulate temperature, which varied between 27 and $29^{\circ} \mathrm{C}$ [ 80 and $85^{\circ} \mathrm{F}$ ]). All combinations of eggs and sperm resulted in fertilization of more than $90 \%$ of mature eggs, as estimated by the observation of fertilization membranes with the dissecting microscope. By $15 \mathrm{hr}$ after fertilization a mixture of swimming blastulas and very early gastrulas (with the archenterons just starting to invaginate) was present in all cultures. By $22 \mathrm{hr}$ all cultures were in the early gastrula stage (archenterons one-third to one-half the length of the blastocoel), and by $38 \mathrm{hr}$ the midgastrula stage had been reached, with the tip of the archenterons expanded but with no coelomes formed. Between 48 and $72 \mathrm{hr}$ the coelomes had formed in all cultures. Thus the reciprocal crosses of the two types of L. multifora demonstrated the same morphology and rate of development as the cultures in which the eggs were fertilized with the same kind of sperm. The cultures terminated at the swimming larval stage because we were not able to provide adequate conditions for further development. Using different parents, we performed the blue gray control crosses and the blue gray female $\times$ red male crosses on another occasion with similar results.

To determine the proportion of each type of L. multifora that was capable of sexual reproduction in early December, individuals of different sizes were dissected or sectioned to determine if gonads containing mature gametes were present. Nine blue gray reef-top L. multifora ranging in size from 3.5 to $5 \mathrm{~cm}$ along the longest arm were dissected, and all but one contained ripe gonads. Of the seven blue gray specimens that were sectioned, six that ranged in size from 2.6 to $3.9 \mathrm{~cm}$ were ripe, and only one $(2.9 \mathrm{~cm})$ was not ripe. Thus $88 \%$ of the specimens examined were ripe in December. Although we were unable to examine any specimens from the smallest size class $(2.5 \mathrm{~cm}$ or less) seen in Figure $1 \mathrm{~A}$, this size range makes up only $13 \%$ of the total. Even if none of these was ripe, at least $76 \%$ of the blue gray specimens as shown in Figure $1 A$ would be expected to be ripe in early December.

Seventeen of the red, subtidal specimens collected in early December were examined by dissection, and an additional six were sectioned. The smallest specimen examined (arm length $1.3 \mathrm{~cm}$ ) was not ripe. Of the 10 specimens with an arm 2.3 to $3.6 \mathrm{~cm}$ in length, $60 \%$ were ripe. The 12 specimens that had an arm $3.7 \mathrm{~cm}$ or longer were all ripe. When these data were compared with the size distribution data from Bernie's Reef, which showed $19 / 62$ specimens with the longest arm less than $2.2 \mathrm{~cm}$ in length, 38 in the $2.3-$ to $3.6-$ $\mathrm{cm}$ size range, and 5 larger than $3.6 \mathrm{~cm}$, it was calculated that $45 \%$ of the specimens shown in Figure $1 B$ could be expected to be ripe. In the case of the data from other underwater reefs (Figure 1C), 73/147 were in the smallest category (of which none was expected to be ripe), 68 were in the medium category (of which $60 \%$ would be expected to be ripe), and all 6 in the largest category would be expected to be ripe, giving an estimate that $32 \%$ of the entire group would be mature.

Observation of specimens held in aquarium tanks for up to $1 \mathrm{yr}$ showed that the two distinctive colors were maintained. When a specimen autotomized an arm, we noticed that it was always one of the largest arms that was removed, confirming the observation of Rideout (1978).

Sequencing of the mitochondrial gene for cytochrome oxidase subunit I was performed to confirm the identifications of the two types of L. multifora and to examine the relationship between them. Phylogenetic trees (Figures 2 and 3) that included sequences from L. guildingi (AF187937), L columbiea (AF187935), and Fromia indica (AF187948) showed that all of the Lm Raro sequences (Genbank numbers DQ451832DQ451839) clustered with L. multifora and L. laevigata (Genbank numbers AF187892AF187921 plus AJ493187-AJ493190) and were not more closely related to the sequences of any other species of Linckia or other members of the family Ophidiasteridae. 


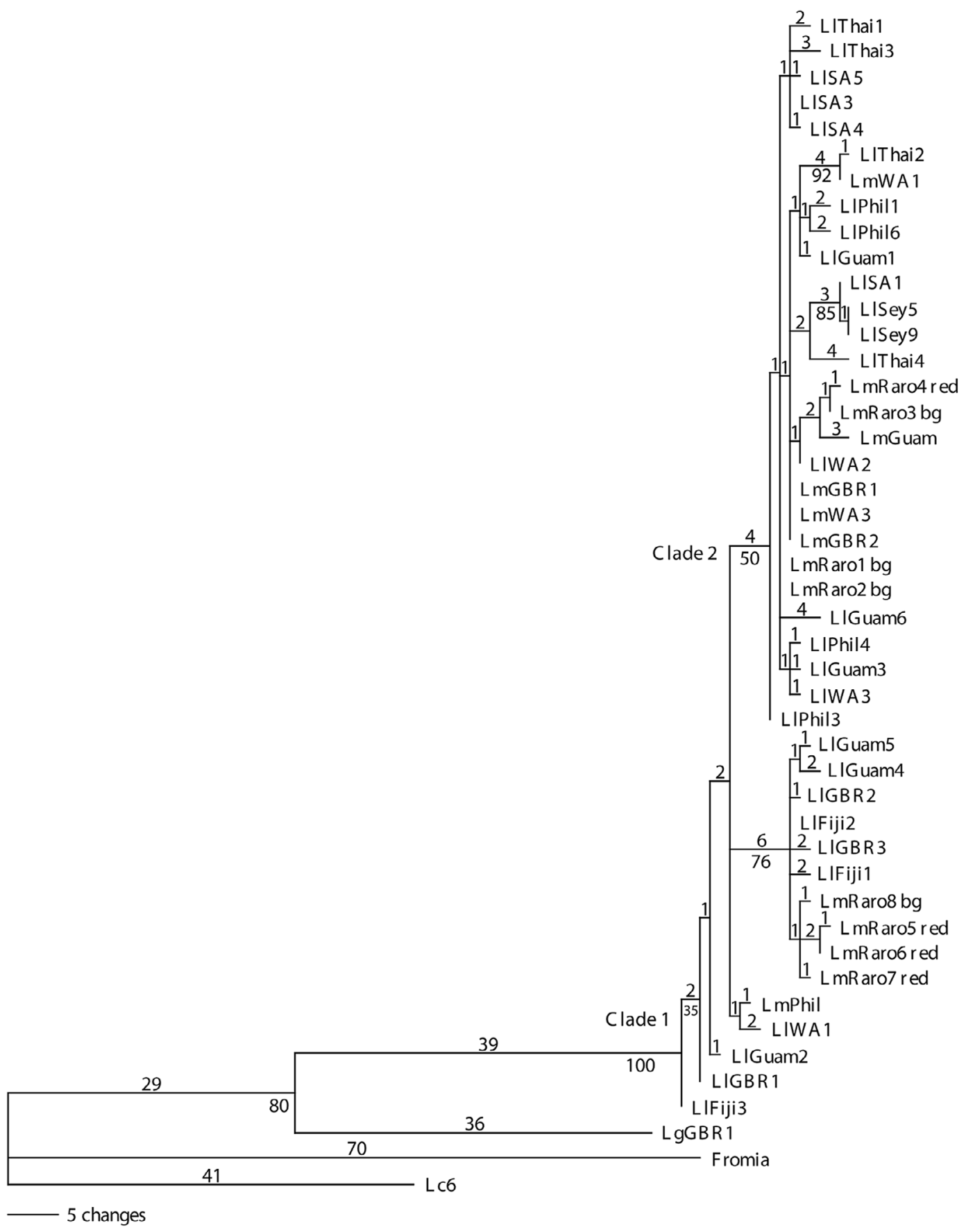

FIgURE 2. Phylogenetic tree based on Maximum-Parsimony analysis of COI sequences from specimens of Linckia multifora from Rarotonga (Lm Raro) (Genbank numbers DQ451832 to DQ451839) with sequences from Williams (2000) of Linckia laevigata (Ll) and Linckia multifora (Lm) from other localities (Genbank numbers AF187892AF187921 plus AJ493187-AJ493190). The numbers above the branches are the base pair differences, and the bootstrap values (1,000 replicates) are shown below the branches. The Rarotonga Lm samples are identified as either from the intertidal (bg) or from the subtidal (red) locations. 


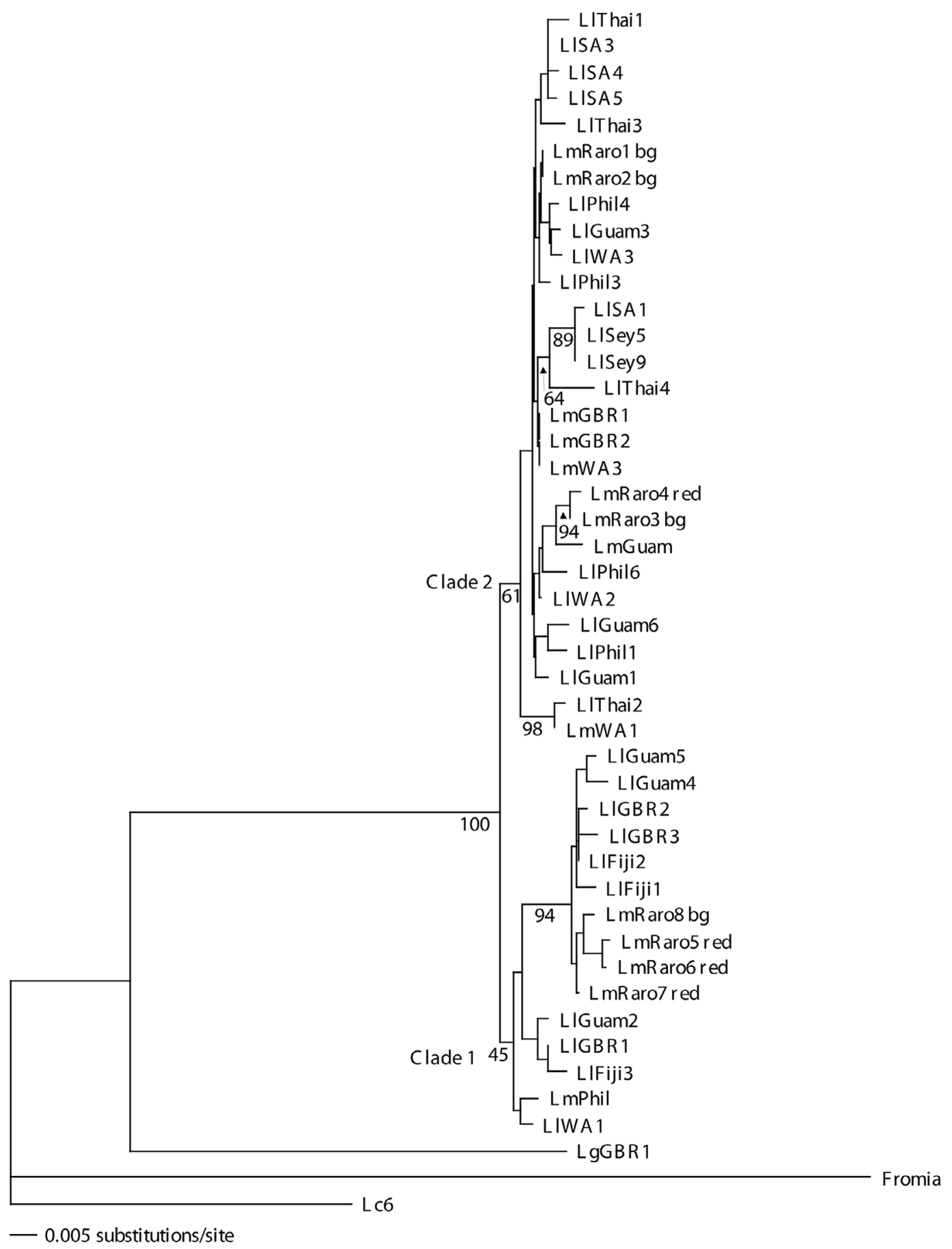

Figure 3. Phylogenetic tree based on Neighbor Joining analysis of COI sequences as described in Figure 2. 

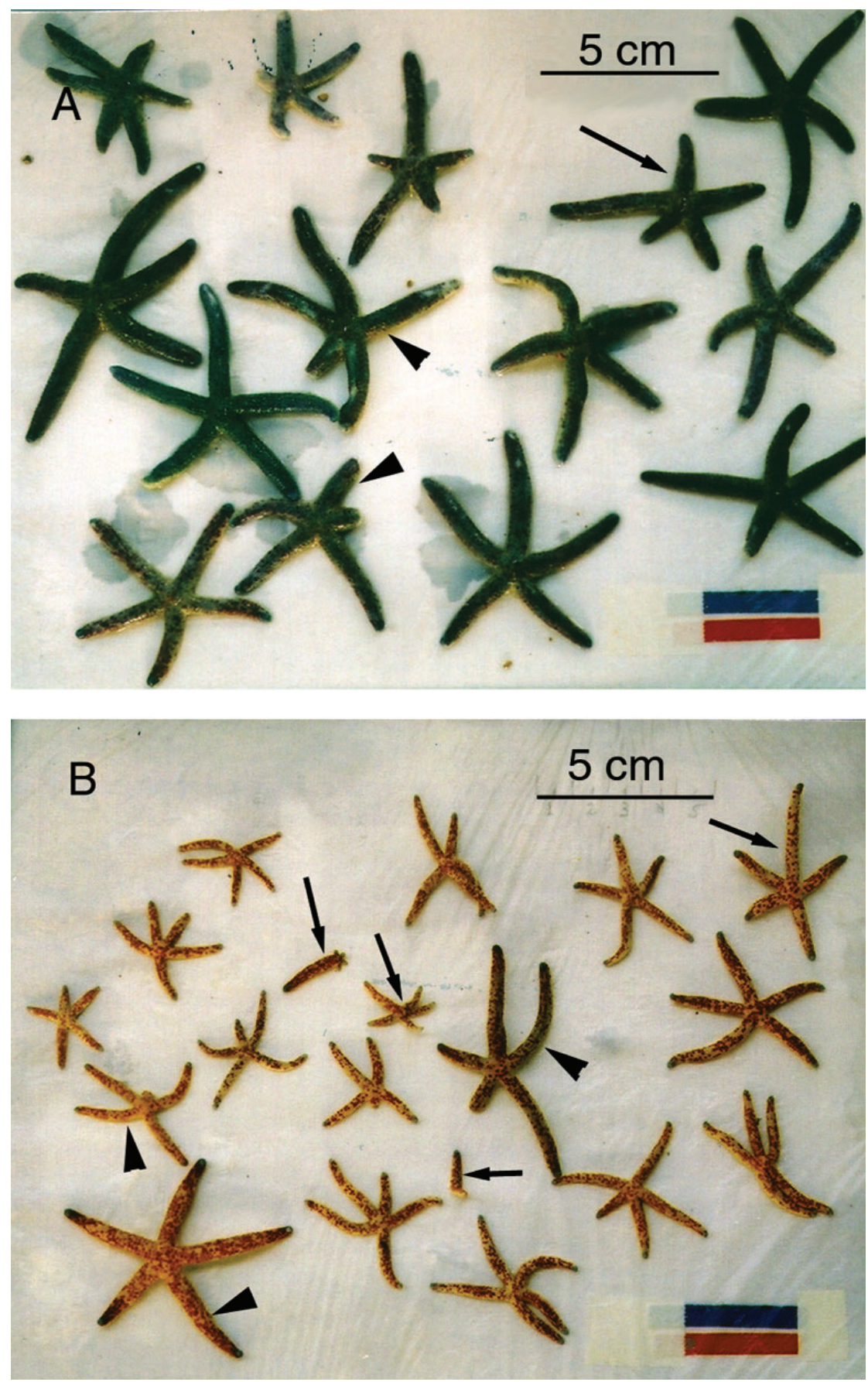

Plate I. Linckia multifora from Rarotonga. A, Specimens collected from intertidal location (reef top). $B$, Specimens collected from subtidal location. Comets (arrows) and counter-comets (arrowheads) can be seen in both groups. Note that the intertidal animals $(A)$ are generally larger and have thicker arms relative to length than the subtidal ones $(B)$. 
TABLE 1

Comparison of Mitochondrial COI Haplotypes of Linckia multifora (Lm) of Rarotonga with Haplotypes of Lm and Ll (L. laevigata) from Australia (WA) and Fiji

\begin{tabular}{|c|c|c|c|c|c|c|c|c|c|c|c|c|c|c|c|}
\hline Haplotype & 56 & 182 & 185 & 194 & 206 & & & 298 & 352 & 408 & 430 & 508 & Phenotype & $\begin{array}{l}\text { Haplotype } \\
\text { Category }\end{array}$ & $\begin{array}{c}\text { GenBank } \\
\text { No. }\end{array}$ \\
\hline Lm WA3 & $\mathrm{T}$ & $\mathrm{T}$ & A & A & $\mathrm{C}$ & $\mathrm{T}$ & $\mathrm{C}$ & G & A & $\mathrm{C}$ & A & $\mathrm{T}$ & & & AF187929 \\
\hline aro 1 (bg) & $\mathrm{T}$ & $\mathrm{T}$ & $\mathrm{A}$ & $\mathrm{A}$ & $\mathrm{C}$ & $\mathrm{T}$ & $\mathrm{C}$ & G & A & $\mathrm{C}$ & A & $\mathrm{T}$ & gray, reef top & Clade 2 & 1832 \\
\hline Lm Raro 2 & $\mathrm{~T}$ & $\mathrm{~T}$ & $\mathrm{~A}$ & $\mathrm{~A}$ & $\mathrm{C}$ & $\mathrm{T}$ & $\mathrm{C}$ & G & A & $\mathrm{C}$ & $\mathrm{A}$ & $\mathrm{T}$ & & & \\
\hline & $\mathrm{T}$ & $\mathrm{T}$ & A & A & $\mathrm{C}$ & $\mathrm{T}$ & $\mathrm{C}$ & G & A & $\mathrm{C}$ & $\mathrm{A}$ & $\mathrm{T}$ & & & \\
\hline & $\mathrm{T}$ & $\mathrm{T}$ & $\mathrm{A}$ & $\mathrm{A}$ & $\mathrm{C}$ & $\mathrm{T}$ & $\mathrm{C}$ & G & A & $\mathrm{C}$ & $\mathrm{A}$ & $\mathrm{T}$ & & $\mathrm{Cla}$ & 335 \\
\hline & $\mathrm{T}$ & $\mathrm{T}$ & A & A & $\mathrm{C}$ & $\mathrm{T}$ & $\mathrm{C}$ & G & A & $\mathrm{C}$ & A & $\mathrm{T}$ & & & \\
\hline & $\underline{\mathrm{C}}$ & $\underline{\mathrm{C}}$ & $\underline{\mathrm{G}}$ & $\underline{\mathrm{G}}$ & $\underline{\mathrm{A}}$ & $\underline{\mathrm{C}}$ & $\underline{T}$ & $\underline{\mathrm{A}}$ & $\underline{\mathrm{G}}$ & $\underline{T}$ & $\underline{\mathrm{C}}$ & $\underline{\mathrm{C}}$ & red, & & DQ4 \\
\hline & $\overline{\mathrm{C}}$ & $\overline{\mathrm{C}}$ & $\overline{\mathrm{G}}$ & $\overline{\mathrm{G}}$ & $\underline{\overline{\mathrm{A}}}$ & $\overline{\mathrm{C}}$ & $\overline{\mathrm{T}}$ & $\underline{\overline{\mathrm{A}}}$ & $\overline{\mathrm{G}}$ & $\overline{\mathrm{T}}$ & $\underline{\overline{\mathrm{G}}}$ & $\overline{\mathrm{C}}$ & red, & & 1837 \\
\hline Lm Raro 7 (red) & $\overline{\mathrm{C}}$ & $\overline{\mathrm{C}}$ & $\overline{\mathrm{G}}$ & $\underline{\mathrm{G}}$ & $\underline{\overline{\mathrm{A}}}$ & $\underline{\mathrm{C}}$ & $\underline{\bar{T}}$ & $\underline{\mathrm{A}}$ & $\underline{\mathrm{G}}$ & $\underline{\mathrm{T}}$ & $\underline{\mathrm{G}}$ & $\underline{\bar{C}}$ & red, subtidal & Clad & DQ451838 \\
\hline$\overline{\text { Lm Raro } 8 \text { (bg) }}$ & $\underline{\bar{C}}$ & $\underline{\overline{\mathrm{C}}}$ & $\underline{\bar{G}}$ & $\underline{\overline{\mathrm{G}}}$ & $\underline{\overline{\mathrm{A}}}$ & $\underline{\bar{C}}$ & $\underline{\bar{T}}$ & $\underline{\overline{\mathrm{A}}}$ & $\underline{\bar{G}}$ & $\underline{\overline{\mathrm{T}}}$ & $\underline{\bar{G}}$ & $\underline{\bar{C}}$ & gray, reef top & $\overline{\text { Clade } 1}$ & DQ451839 \\
\hline Lm Raro 9 (red) & $\overline{\mathrm{C}}$ & $\underline{\bar{C}}$ & $\overline{\mathrm{G}}$ & $\overline{\mathrm{G}}$ & $\underline{\bar{A}}$ & $\underline{\bar{C}}$ & $\overline{\mathrm{T}}$ & $\bar{?}$ & $\overline{\mathrm{G}}$ & $\overline{\mathrm{T}}$ & $\overline{\mathrm{G}}$ & $\overline{\mathrm{C}}$ & red, subtidal & & \\
\hline Ll Fiji 2 & $\underline{\overline{\mathrm{C}}}$ & $\underline{\bar{C}}$ & $\underline{\overline{\mathrm{G}}}$ & $\underline{\bar{G}}$ & $\underline{\bar{A}}$ & $\underline{\overline{\mathrm{C}}}$ & $\underline{\bar{T}}$ & $\underline{\overline{\mathrm{A}}}$ & $\underline{\overline{\mathrm{A}}}$ & $\underline{\bar{T}}$ & $\underline{\bar{G}}$ & $\underline{\bar{C}}$ & & & $\underline{\mathrm{AF} 187895}$ \\
\hline
\end{tabular}

Note: The base position is given relative to Lm WA2. Underlining indicates the bases shared by the Clade 1 haplotype class and the Ll Fiji 2 haplotype. Lm Raro 9 and Lm Raro 10 sequences were derived from a single clone each and therefore were not used for the phylogenetic tree or submitted to GenBank.

Figure 2 shows that haplotypes of COI from both types of L. multifora from Rarotonga clustered within the two clades of $L$. multifora and L. laevigata from other localities. COI LmRaro sequences from the two clades exhibit 12 fixed differences (Table 1). Both the Parsimony and Neighbor Joining trees (Figures 2 and 3) show that Lm Raro 1, 2, 3, and 4 fall into Clade 2 of Linckia as defined by Williams (2000). Internal to the node that defines Clade 2, these fall into two groups of sequences separated from the node by 1 and by 7 base pairs, respectively. A distance of 4 base pairs separates the two clades, and a further 6 base pairs within Clade 1 leads to an association of Lm Raro 5-8 with haplotypes from Australia, Fiji, and Guam. Sequences from both the red, subtidal type of L. multifora from Rarotonga and the blue gray intertidal form were found in both clades.

\section{DISCUSSION}

In this study we have described the presence of two morphotypes of Linckia multifora that are found in different environments on Rarotonga, and we have studied the mechanisms of reproduction that they use. Linckia multifora has often been assumed to reproduce pri- marily or only by asexual means through the process of autotomy. This occurs when a parent animal autotomizes an arm that then regenerates four arms (comet stage) while the parent (counter-comet) regenerates the autotomized arm. Rideout (1978) felt that sexual reproduction probably was a sporadic phenomenon in this species and that populations on Guam successfully maintain themselves through asexual reproduction. However, photographs of samples taken at various sites in Rarotonga suggested to us that here, at least, sexual reproduction must be common. If only asexual reproduction occurred, we would expect that all of the comets would be regenerating from arms of the largest size. Instead we found that the individuals covered a whole range of sizes and that comets were regenerating from both very tiny (as small as $0.7 \mathrm{~cm}$ ) as well as larger arms (up to ca. $5 \mathrm{~cm}$ ) (Plate I). The very small arms come from very small (young) parents, because individuals always lose their largest arm to autotomy. This type of size distribution suggests that the population consists of individuals of a whole variety of ages since metamorphosis from the larval stage. Although some variation in size can occur in starfish by shrinkage, individuals in these populations did not appear to be starv- 
ing because they were turgid in appearance; in our experience, individuals that did not eat while being kept in the aquarium became somewhat flacid. As shown by dissection and sectioning of specimens, there is a high proportion of sexually mature individuals in the red, subtidal group (32-45\%) and the blue gray intertidal group (76\%). At the subtidal sites there is a greater abundance of small specimens (Figure 1), and the median size of the smallest specimens is $1 \mathrm{~cm}$ less than those at the intertidal site. At the intertidal site the individuals are larger, and therefore a greater proportion of the individuals are capable of reproducing sexually as well as asexually.

The sequencing data strongly suggest that the two morphological types of L. multifora in Rarotonga form a single interbreeding population. Each group contains the same two classes of haplotypes of COI (Table 1) with no consistent differences between them. If these two types of L. multifora were reproductively isolated we would expect that they would have accumulated different sets of random mutations in the COI gene (Avise 2000). The occurrence of equally successful fertilization and early development for the four combinations of eggs and sperm from these two types also suggests that the two populations are capable of interbreeding. However, due to lack of facilities and methods to allow later development and metamorphosis we cannot be certain that development of all combinations can continue to adulthood.

The most likely explanation of these observations is that sexual reproduction of $L$. multifora in Rarotonga occurs in the breeding season (which includes the period from late November to early December) and, after entering the plankton, the larvae may preferentially settle at the Bernie's Reef site or other subtidal sites. This would account for the larger proportion of individuals of the smaller sizes found at the subtidal sites as well as the very large total number found at the Bernie's Reef site. From here the smallest adults may be dispersed to various other sites by the waves and currents, or they may migrate toward the shallower areas, and those arriving on the reef top may be triggered to develop additional blue gray pigment as a protection from excessive sunlight, which has also been suggested to occur in L. laevigata that are found in shallow waters (Williams 2000). In Rarotonga larval recruitment of $L$. multifora may also occur directly at all of the sites, and our lack of observation of very small specimens (less than $1.9 \mathrm{~cm}$ in the length of the longest arm) at the intertidal site may be due to the difficulty of seeing them in this environment. In addition, at both the intertidal and subtidal sites, autotomy of arms followed by regeneration must be very important in maintaining the populations, as shown by the substantial proportion of individuals that can be classified as comets (having one arm larger than the others) of $10-15 \%$ at all sites. The presence of comets shows that autotomized arms have successfully developed into new individuals.

Sexual reproduction in L. multifora had not been examined since Mortenson (1938) described fertilization and development of a very few eggs in culture. It is now known that to obtain a high proportion of fertilizable starfish eggs it is necessary to treat them with a maturation factor such as nerve extract or 1-methyl adenine (Stevens 1970, Kanatani 1973). Rideout (1978), who studied the $L$. multifora population in Guam, found that eggs were released from one specimen when injected with 1-methyl adenine but concluded that the population in Guam was maintained primarily by asexual reproduction. Our findings that a high proportion of both male and female specimens collected from the field had mature gonads during late November and early December and that fertilization and early development could be accomplished in the laboratory suggest that sexual reproduction probably occurs in nature. In addition, the presence of two distinctive types of COI sequences in both the reef-top and the subtidal forms of L. multifora strongly suggests that sexual reproduction, which would result in gene flow between different areas, has occurred.

Many observations have been made of cryptic sympatric species or subspecies of coral reef organisms (reviewed by Knowlton 1993). The two types of COI sequences found in the L. multifora of Rarotonga differ 
in 12 base positions, amounting to a $2.3 \%$ difference in the 524 base pair sequence. This is close to the lower limit 2.5-24.2 difference for COI found between nominal Asterinidae species (Hart et al. 1997), suggesting that there may be two cryptic subspecies or species within the L. multifora in Rarotonga. However, the accumulating evidence for sporadic gene flow rather than continuous gene mixing throughout the Pacific (Benzie 1999) can explain the apparent existence of cryptic sympatric species or subspecies in some coral reef organisms, such as in L. guildingi (Williams 2000) as well as in L. multifora in Rarotonga. A dual origin of L. multifora of Rarotonga is suggested by the COI sequencing data, which did not reveal any intermediates between the two categories of COI sequences having the 12 fixed differences.

The existence of the two different morphological types of Linckia multifora on Rarotonga cannot be explained solely by the existence of two founding groups because the two classes of haplotypes of COI are found in both morphological types. One possible explanation is that each morphological type had a different geographical origin, but mitochondrial introgression has blurred the (sub)species boundaries. Another possible explanation for the two morphological types is that they are ecophenotypes (i.e., their permanent differences have been triggered by environmental effects during development). This phenomenon has been reported in plants and colonial invertebrates (reviewed by Appleton and Palmer 1988) as well as in some solitary mollusks (Appleton and Palmer 1988, Espoz et al. 2004). Williams (2000) invoked this mechanism to explain the observation that $L$. multifora and L. laevigata cannot be distinguished on the bases of COI sequences and therefore may actually be ecophenotypes of the same species. The existence of ecophenotypes is probably the simplest explanation for the morphological differences between the intertidal and subtidal groups of L. multifora on Rarotonga, because the formation of additional dark pigment may protect against the effects of sunlight on the intertidal organisms. In future it may be possible to determine if the mor- phological differences between the two types of L. multifora (and also L. laevigata) are a result of genetic differences or environmental effects if their development can be followed in the laboratory through metamorphosis and beyond.

\section{ACKNOWLEDGMENTS}

We thank Barry Hill and Eric Bateman of Dive Rarotonga for their assistance with collections and Michael Chan for assistance with PCR. We thank three anonymous reviewers for their comments, which have greatly improved this work.

\section{Literature Cited}

Appleton, R. D., and A. R. Palmer. 1988. Water-born stimuli released by predatory crabs and damaged prey induce more predator-resistant shells in a marine gastropod. Proc. Natl. Acad. Sci. U.S.A. 85:4387-4391.

Avise, J. C. 2000. Phylogeography: The history and formation of species. Harvard University Press, Cambridge, Massachusetts.

Benzie, J. A. H. 1999. Genetic structure of coral reef organisms: Ghosts of dispersal past. Am. Zool. 39:131-145.

Clark, A. C., and F. Rowe. 1971. Monograph of shallow-water Indo-Pacific echinoderms. Br. Mus. (Nat. Hist.) Spec. Publ. 690:1-238.

Ebert, T. A. 1996. The consequences of broadcasting, brooding, and asexual reproduction in echinoderm metapopulations. Oceanol. Acta 19:217-226.

Edmondson, C. H. 1935. Autotomy and regeneration in Hawaiian starfishes. Occas. Pap. Bernice Pauahi Bishop Mus. 11:320.

Espoz, C., D. R. Lindberg, J. C. Castilla, and W. B. Simison. 2004. Intertidal limpets of Chile and Peru. Rev. Chil. Hist. Nat. 77:257-283.

Hart, M. W. 2002. Life history evolution and comparative developmental biology of echinoderms. Evol. Dev. 4:62-71.

Hart, M. W., M. Byrne, and M. J. Smith. 
1997. Molecular phylogenetic analysis of life history evolution in asterinid starfish. Evolution 51:1848-1861.

Kanatani, H. 1973. Maturation inducing substances in starfish. Int. Rev. Cytol. 35:253298.

Knowlton, N. 1993. Sibling species in the sea. Annu. Rev. Ecol. Syst. 24:189-216.

Marsh, L. M. 1974. Shallow-water asterozoans of southeastern Polynesia I. Asteroidea. Micronesica 10:65.

Mortensen, T. 1938. Contributions to the study of the development and larval forms of echinoderms. IV. K. Dan. Vidensk. Selsk. Skr. (Naturv. Math.) 9:1-59.

Rideout, R. S. 1978. Asexual reproduction as a means of population maintenance in the coral reef asteroid Linckia multifora on Guam. Mar. Biol. (Berl.) 47:287-295.
Stevens, M. 1970. Procedures for induction of spawning and induction of maturation of starfish oocytes by treatment with 1methyladenine. Exp. Cell Res. 59:482484.

Swofford, D. L. 2003. PAUP: Phylogenetic analysis using parsimony (and other methods). Version 4.0d81. Sinauer Associates, Sunderland, Massachusetts.

Williams, S. T. 2000. Species boundaries in the starfish genus Linckia. Mar. Biol. (Berl.) 136:137-148.

Yamaguchi, M. 1977. Population structure, spawning and growth of the coral reef asteroid Linckia laevigata (Linnaeus). Pac. Sci. 31:13-30.

Zyskind, J. W., and S. I. Bernstein. 1989. Recombinant DNA laboratory manual. Academic Press, San Diego. 
